\title{
Rationale and design of the RIBS IV randomised clinical trial (drug-eluting balloons versus everolimus-eluting stents for patients with drug-eluting stent restenosis)
}

\author{
Fernando Alfonso $^{1 *}$, MD; María José Pérez-Vizcayno², MD; Alberto Cárdenas², MD; \\ Bruno García del Blanco ${ }^{3}$, MD; Arturo García-Touchard ${ }^{4}$, MD; José Ramón López-Minguéz ${ }^{5}$, MD; \\ Fernando Rivero $^{1}$, MD; Mónica Masotti ${ }^{6}$, MD; Javier Zueco ${ }^{7}$, MD; Angel Cequier ${ }^{8}$, MD; Cesar Morís ${ }^{9}$, MD; $^{2}$ \\ Antonio Fernández-Ortíz ${ }^{2}$, MD; Javier Escaned², MD; Pilar Jiménez-Quevedo ${ }^{2}$, MD; Nieves Gonzalo², MD; \\ Cristina Fernández ${ }^{2}$, MD; Carlos Macaya², MD; for the Restenosis Intra-stent: drug-eluting Balloon vs. \\ everolimus-eluting Stent (RIBS IV) Study Investigators (under the auspices of the Working Group on \\ Interventional Cardiology of the Spanish Society of Cardiology)
}

\section{Hospital Universitario de La Princesa, Madrid, Spain; 2. Hospital Universitario Clínico San Carlos, Madrid, Spain; \\ 3. Hospital Universitario Vall d'Hebron, Barcelona, Spain; 4. Hospital Universitario Puerta de Hierro-Majadahonda, Madrid, \\ Spain; 5. Hospital Universitario Infanta Cristina, Badajoz, Spain; 6. Hospital Universitario Clinic de Barcelona, Barcelona, \\ Spain; 7. Hospital Universitario Marqués de Valdecilla, Santander, Spain; 8. Hospital Universitario de Bellvitge, Barcelona, \\ Spain; 9. Hospital Universitario Central de Asturias, Oviedo, Spain}

This paper also includes accompanying supplementary data published online at: http://www.pcronline.com/eurointervention/86th_issue/61

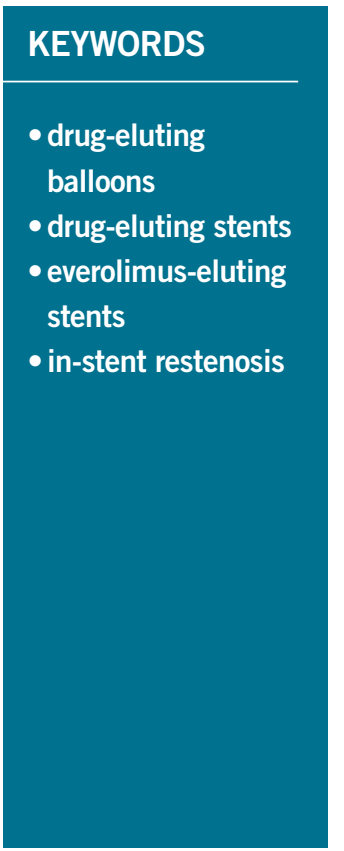

\begin{abstract}
Aims: This study will compare the efficacy of drug-eluting balloons (DEB) and everolimus-eluting stents (EES) in patients with drug-eluting stent (DES) in-stent restenosis (ISR).

Methods and results: This is a prospective, multicentre, randomised clinical trial comparing DEB and EES in patients with DES-ISR. The study is an investigator-driven initiative generated within the RIBS study programme. A total of 310 patients with DES-ISR will be included and randomised $(1: 1)$ to DEB or EES. Angiographic follow-up has been scheduled at six to nine months. Quantitative coronary analyses will be performed in a centralised core lab by blinded personnel. The primary endpoint of the study is minimal lumen diameter at angiographic follow-up. Other secondary angiographic endpoints include \% diameter stenosis, late loss, net gain and binary restenosis rate. An independent clinical events committee will adjudicate clinical events after reviewing source documents. The main clinical outcome measure is a combined endpoint of cardiac death, myocardial infarction and target vessel revascularisation at one year. Individual components of the combined clinical endpoint and rates of target lesion revascularisation and stent thrombosis will also be compared.
\end{abstract}

Conclusions: This randomised clinical trial will determine the relative efficacy of EES versus DEB in patients presenting with DES-ISR. (ClinicalTrials.gov Identifier: NCT01239940).

\footnotetext{
*Corresponding author: Departamento de Cardiología, Hospital Universitario de La Princesa, IIS-IP, Universidad Autónoma de Madrid, c/ Diego de León 62, 28006 Madrid, Spain. E-mail: falf@hotmail.com
} 


\section{Introduction}

Drug-eluting stents (DES) are widely used during coronary interventions $^{1,2}$. DES reduce neointimal proliferation and the need for repeated revascularisation ${ }^{1,2}$. However, DES are not immune to in-stent restenosis (ISR) ${ }^{3,4}$. Although rare, DES-ISR represents a significant clinical problem ${ }^{3,4}$. Notably, reinterventions in patients with DES-ISR are associated with poorer outcomes as compared with those found in patients treated for bare metal stent (BMS) ISR $^{3-7}$. Although the therapy of choice for patients with DES-ISR still remains debatable ${ }^{3}$, DES implantation is frequently recommended in this setting ${ }^{7-10}$. More recently, however, drug-eluting balloons (DEB) have also demonstrated favourable late outcomes in patients with BMS-ISR and DES-ISR ${ }^{11-18}$. In this scenario, the results of DEB appear to be similar to those obtained with firstgeneration $\mathrm{DES}^{11-18}$.

New-generation DES are safer and more effective than first-generation DES ${ }^{19,20}$. Furthermore, the value of second-generation DES has also been demonstrated in complex lesion subsets, including ISR $^{21}$. However, the relative efficacy of second-generation DES, as compared to DEB, in patients with DES-ISR remains unknown. In this randomised trial we will compare the results of EES versus DEB in patients with DES-ISR.

\section{Methods STUDY PROTOCOL}

The Restenosis Intra-stent: drug-eluting Balloon vs everolimus-eluting Stent (RIBS IV) study is a multicentre, prospective, open-label, controlled, randomised clinical trial which compares the results of EES versus DEB in patients with DES-ISR (ClinicalTrials.gov Identifier: NCT01239940) (Online Appendix). Inclusion/exclusion criteria are largely similar to those used in previous RIBS trials ${ }^{7,8}$. Patients $>18$ years of age presenting with angina or ischaemia and showing DES-ISR ( $>50 \%$ diameter stenosis on visual assessment) on coronary angiography are eligible ${ }^{7,8}$. Patients in whom the stent type cannot be established, and those with stent location unclear on angiography are excluded. Patients with DES-ISR on small vessels (diameter $\leq 2.0 \mathrm{~mm}$ ) and those with very diffuse lesions (length $>30 \mathrm{~mm}$ ) or total occlusions (TIMI 0), are also excluded ${ }^{7,8}$. However, patients with $>1$ episode of ISR and those with $>1$ stent layer at the target lesion are eligible. To avoid including patients with stent thrombosis, patients with very early ( $<1$ month) ISR, and those presenting with an acute myocardial infarction (criteria below) or angiographic thrombus, are not included ${ }^{7,8}$. However, patients with unstable symptoms presenting with troponin elevation, but with normal creatine-kinase levels, are eligible. Patients with edge-ISR will be eligible when involvement of the stent border is confirmed (confined to the edge or extending into the adjacent coronary segment). In these cases, multiple angiographic views and recording of the stent on fluoroscopy prior to the injection of dye are recommended to clarify the relationship between the stenosis and the stent edge. The use of intracoronary imaging techniques to confirm stent edge involvement is recommended. Patients with severe systemic illnesses (including renal and hepatic) or a life expectancy
$<1$ year are excluded. Finally, patients with severe peripheral vascular disease potentially interfering with angiographic follow-up are not eligible ${ }^{7,8}$. Written informed consent will be obtained from all patients.

Twenty-five university hospitals from Spain have been invited to participate in this trial (Online Appendix). Telephonic randomisation (1:1) will be performed at the coordinating centre using a computer-generated sequence ${ }^{7,8}$. Randomisation will be stratified according to ISR length ( $\leq 10$ vs. $>10 \mathrm{~mm}$ ) and location (edge-ISR vs. intra-stent ISR) on visual analysis.

On-site monitoring and data assessment, collection, management and analysis will be organised by and performed at the coordinating centre (Clínico San Carlos University Hospital, Madrid, Spain). The study is an investigator-driven initiative organised and conducted under the auspices of the Working Group on Interventional Cardiology of the Spanish Society of Cardiology. Unrestricted research grants have been obtained from B. Braun Surgical and Abbott Vascular. The study protocol is in accordance with the provisions of the Declaration of Helsinki and needs to be approved by the institutional ethics committees of all participating sites.

\section{PROCEDURES}

All patients will be pretreated with aspirin and clopidogrel. Procedures will be performed under full anticoagulation with unfractionated heparin. The protocol mandates careful and complete lesion predilation. Initially, lesions should be predilated with relatively short balloons and low pressures to ensure balloon stabilisation at the lesion site and to avoid any damage to the adjacent segments. If required, buddy wire techniques or cutting balloons will be used to avoid balloon slippage or watermelon seeding phenomena. Then, the systematic use of high pressures is recommended. However, great attention will be paid to identify and tackle underexpanded stents. In this scenario, the protocol recommends the use of short non-compliant balloons at very high pressures ${ }^{7,8,21}$. Once adequate lesion predilation has been achieved, patients will receive the allocated treatment. EES (XIENCE PRIMETM; Abbott Vascular, Abbott Park, IL, USA) will be implanted using a 1:1:1 final balloon-to-artery ratio and relatively high deployment pressures ( $>14$ bar). After EES implantation, post-dilation with non-compliant balloons is recommended but will be performed at the discretion of the operator. However, high-pressure post-dilation with non-compliant balloons is mandated for patients with suboptimal angiographic results and also for those with underexpanded stents on fluoroscopy. Great care will be taken to ensure that all these highpressure balloon inflations are performed within the stented segment. Alternatively, in patients allocated to a DEB, after lesion predilation, DEB (SeQuent ${ }^{\circledR}$ Please; B.Braun Melsungen AG, Melsungen, Germany) will be used, selecting a 1:1:1 balloon-to artery ratio and nominal pressures (12-14 atm) for 60 seconds. Crossover to bail-out stenting is strongly discouraged but allowed in cases with dilation failure ( $>50 \%$ residual stenosis) or major ( $\geq$ type $C$ ) edge dissections.

The protocol suggests the use of intracoronary imaging techniques (intravascular ultrasound or optical coherence tomography) to assess neointimal tissue and stent expansion, and to optimise 
procedural results. However, the selection of these imaging techniques and the criteria selected to guide final optimisation strategies will be left to the operator's decision. Patients treated under the guidance of these techniques will be entered into the corresponding imaging substudies.

Serial enzymatic determinations, including serum creatinekinase (with MB band analyses when abnormal) and troponin levels will be obtained. In addition, serial 12-lead electrocardiograms will be obtained every eight hours for the first 24 hours $^{7,8}$. Clopidogrel (75 mg/day) is recommended for one year after EES implantation, and for three months after DEB therapy. Aspirin will be used indefinitely.

\section{DEFINITIONS AND CLINICAL AND ANGIOGRAPHIC FOLLOW-UP}

Patients will be followed up at six to nine months, one year, and then annually for five years. Angiographic follow-up will be scheduled at six to nine months, but this examination will be performed earlier if clinically indicated. Electronic case report forms will be completed at each site by local investigators, monitored and submitted to the coordinating centre. At the coordinating centre, data will be critically reviewed for completeness and consistency. When required, specific queries will be sent to the sites concerned. All the data will be prospectively entered into a dedicated, relational database specifically designed for all the RIBS studies ${ }^{7,8,21}$. Source documents will be obtained from the sites for patients with major adverse events. Clinical events (death, myocardial infarction, target vessel revascularisation) will be adjudicated by an independent clinical events committee. Members of this committee will be blinded to the allocated intervention and will examine the corresponding source documents. Deaths will be considered as cardiac unless a non-cardiac cause is demonstrated. The diagnosis of myocardial infarction has been maintained unchanged in the RIBS trials ${ }^{7,8,21}$. This requires two of the following: a) prolonged ( $>30$ minutes) chest pain, b) rise in creatine-kinase levels greater than twice the local upper normal values (with abnormal MB fraction), and c) development of new ischaemic ECG changes (with or without development of pathological Q-waves). All repeated interventions have to be clinically justified (angina or ischaemia documentation). A fractional flow reserve $<0.80$ will also be considered as a clinical indication for revascularisation. Case report forms clearly separate target lesion from target vessel revascularisation. However, all the angiograms of patients requiring target vessel revascularisation will be analysed at the core lab to determine the exact site of revascularisation. The Academic Research Consortium definition will be used to assess stent thrombosis ${ }^{22}$.

\section{ANGIOGRAPHIC ANALYSIS}

All coronary angiograms will be analysed at the central angiographic core laboratory. Studies will be analysed by trained personnel blinded to treatment allocation and using a standard methodology $\mathrm{y}^{7,8,21}$. Lesion morphology will be evaluated using the Mehran classification ${ }^{23}$ and the ACC/AHA ${ }^{24}$ classification.
An automatic edge-detection system (CAAS II System; Pie Medical Imaging BV, Maastricht, The Netherlands) will be used for offline quantitative measurements ${ }^{8,21}$. After intracoronary administration of nitroglycerine, orthogonal views (three separate projections) will be selected by the operator (avoiding vessel foreshortening and the overlap of major side branches) and matched projections will be repeated immediately after the intervention and at late follow-up. Both in-lesion and in-segment (lesion + complete treated segment $+5 \mathrm{~mm}$ adjacent margins) analyses will be performed. The same measurements will be obtained after the procedure and at followup. Results of intravascular ultrasound and optical coherence studies will be analysed in the same central core lab using standard methodology ${ }^{8}$.

\section{MAIN OUTCOME MEASUREMENTS}

The primary angiographic endpoint of the study is the comparison of the in-segment minimal lumen diameter at late follow-up in the two arms. Major secondary angiographic endpoints include percent diameter stenosis, acute gain, net gain, late loss, loss index and binary restenosis rate $(>50 \%$ diameter stenosis). The main clinical outcome measure is a combined endpoint of cardiac death, myocardial infarction and target vessel revascularisation at one year. Individual components of the combined clinical endpoint and rates of target lesion revascularisation and stent thrombosis will also be compared.

\section{STATISTICAL ANALYSES}

Results will be compared with the chi-square test or Fisher's exact test, as required. For continuous variables the Kolmogorov-Smirnov test will be used to determine data distribution. Continuous data will be presented as mean (SD) or median (IQR) and compared using the Student's t-test or the Mann-Whitney test. Main effect estimates will be presented with the corresponding 95\% confidence interval (CI). Kaplan-Meier curves will be constructed to estimate event-free survivals that will be compared with the log-rank and Breslow's exact tests. Hazard ratios (with corresponding 95\% CI) will be assessed using Cox models and compared with the Wald test. The results of main outcome measures will also be examined according to 10 pre-specified variables described in previous RIBS trials ${ }^{7,8,21}$. Formal tests will be used to detect interactions.

Sample size calculation required several assumptions as data on the results of EES/DEB in patients with DES-ISR were not available when the trial was designed. Based on previous RIBS studies, we assumed that a minimal lumen diameter of $2.6 \pm 0.4 \mathrm{~mm}$ will be obtained immediately after stent implantation ${ }^{7,8}$. In patients with BMS-ISR treated with sirolimus-eluting stents, a late loss of $0.13 \mathrm{~mm}$ was obtained in RIBS $\mathrm{II}^{8}$, and of $0.32 \mathrm{~mm}$ in ISARDESIRE $^{9}$. However, in patients with DES-ISR, a greater late loss $(0.4 \mathrm{~mm})$ was reported in ISAR-DESIRE $2^{10}$ using first-generation DES. Assuming the worst case scenario, a late loss of $0.6 \mathrm{~mm}$ after EES was considered. Therefore, a minimal lumen diameter of $2.0 \pm 0.6 \mathrm{~mm}$ was eventually calculated at late follow-up in the EES arm. Likewise, according to the RIBS I and II studies ${ }^{7,8}$, a minimal 
lumen diameter of $2.2 \pm 0.5 \mathrm{~mm}$ was assumed immediately after balloon angioplasty. A previous study of DEB for BMS-ISR showed a late lumen loss of $0.17 \pm 0.42 \mathrm{~mm}^{12}$. However, in patients with DES-ISR, late lumen loss was assumed to be larger after DEB. In a previous mechanistic study, we found an "early lumen loss" of $0.4 \mathrm{~mm}$ after conventional balloon angioplasty ${ }^{25}$. Likewise, a late loss of $0.37 \mathrm{~mm}$ was recently found (but after this trial was designed) in ISAR-DESIRE $3^{17}$. Taking the worst case scenario (late loss of $0.4 \mathrm{~mm}$ ), a minimal lumen diameter at follow-up of $1.80 \pm 0.6$ was assumed in the DEB arm. Using a superiority design with an $80 \%$ power (beta) and an alpha value of 5\%, 142 patients per arm will be required. Compensation for losses to follow-up (estimated 9\%) will require the enrolment of 310 patients (155 per arm). The trial design is presented in Figure 1. This design will be unable to identify differences smaller than $0.2 \mathrm{~mm}$ in minimal lumen diameter at followup, but it is assumed that lower differences will not be clinically relevant. The study is planned as a superiority trial (EES superior to DEB); however, a specific design to demonstrate non-inferiority was not planned. All analyses will be performed according to the intention-to-treat principle unless otherwise specified. The SPSS statistical package will be used. A value of $\mathrm{p}<0.05$ will be considered as statistically significant.

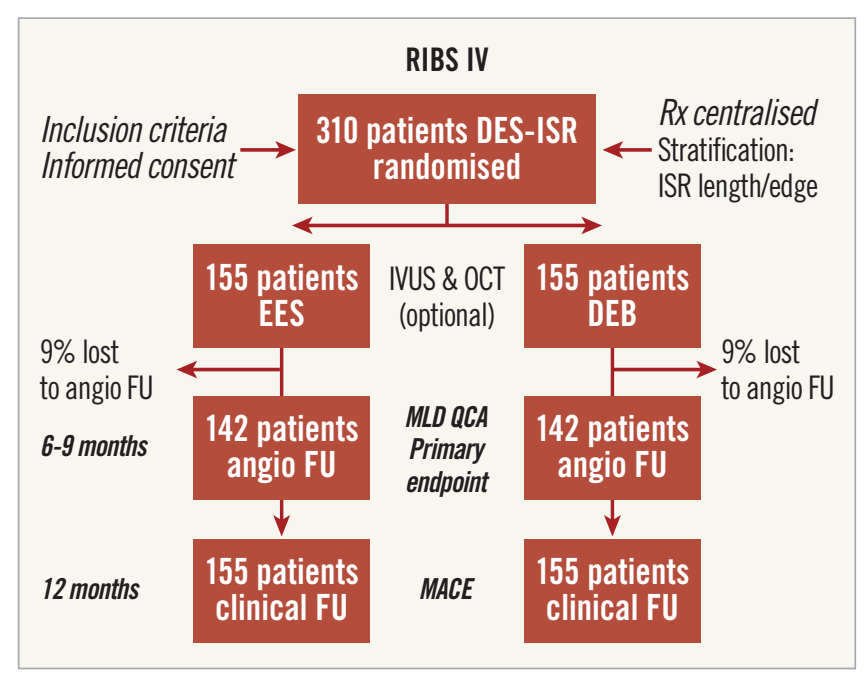

Figure 1. Study flow chart detailing randomisation of patients and patients with late angiography. DEB: drug-eluting balloons; EES: everolimus-eluting stents; FU: follow-up; ISR: in-stent restenosis; MACE: major adverse cardiac events; MLD: minimal lumen diameter; QCA: quantitative coronary angiography

\section{Discussion}

This randomised clinical trial will determine the relative value of EES versus DEB in patients presenting with DES-ISR. Due to the scarce previous data on results in patients with DES-ISR, we selected a pragmatic and conservative sample size calculation and the resultant patient cohort is thus considerably larger than that included in the RIBS V trial. Accordingly, the present study will be powered for important surrogate angiographic endpoints including minimal lumen diameter at follow-up, selected as the primary endpoint. This angiographic outcome measure has been widely used in previous clinical trials comparing coronary interventions using mechanistically different therapeutic strategies. This endpoint is largely preferred over the classic angiographic "late loss" when interventions that provide different acute gains are compared ${ }^{18}$. The comparison of minimal lumen diameter at follow-up will provide an accurate estimate of the relative late efficacy of these competing therapeutic strategies. In accordance with the experience gained in previous RIBS studies, we assumed a larger acute gain after $\mathrm{EES}^{7,8}$. At follow-up, however, we assumed a relatively large late loss in both groups, as treatment of DES-ISR has been consistently associated with relatively poor late angiographic findings ${ }^{5,6,10}$. Our study is powered to detect a difference in minimal lumen diameter $\geq 0.2 \mathrm{~mm}$ in favour of the EES arm. Notably, our study will also be powered for the comparison of other clinically important late angiographic parameters such as percent diameter stenosis, net effective angiographic gain and binary restenosis rates. These major secondary angiographic outcome measures have also been validated previously in trials comparing different devices. These variables will complement the information derived from the analysis of the primary endpoint and will provide valuable additional insights into the relative efficacy of EES and DEB.

Our study, however, is not fully powered to detect differences in clinical outcome measures. However, the assumed difference in minimal lumen diameter at follow-up may well translate into significant differences in target vessel and target lesion revascularisation. These are the main determinants of the combined clinical endpoint which, eventually, might also diverge at one year. However, the selected sample size will be insufficient to detect differences in other major clinical endpoints, including cardiac or total mortality, myocardial infarction and stent thrombosis rates ${ }^{22}$. Nevertheless, this study (involving a total of 310 patients) will still represent a relatively large study on patients with DES-ISR considering the rarity of the condition. Therefore, our results will also provide important safety information. All patients will be followed up for one year and subsequently annually using a detailed predesigned questionnaire.

Last but not least, the results of our pre-specified subgroup analyses will also provide relevant information. Treatment effect may be homogeneous across all major subgroups or, alternatively, potential differences may be detected among subgroups. Notably, however, interaction between these selected variables and the main outcome estimates, if present, will only be considered as hypothesis-generating and will therefore require confirmation in additional studies. These analyses are potentially important as in the future some clinical or angiographic features might eventually be used in selected patients to favour one of these therapeutic modalities over another. For instance, the outcome of patients suffering from a second or third ISR and those having multiple stent layers seems to be worse than that of those patients suffering from first ISR ${ }^{3}$. The results of this challenging cohort of patients will also be analysed in the current study. 


\section{Previous studies of interventions in patients with ISR}

RIBS II $^{8}$ demonstrated that sirolimus DES were superior to conventional balloon angioplasty in patients with BMS-ISR. Likewise, ISAR-DESIRE $1^{9}$ demonstrated that DES are superior to balloon angioplasty in this setting. On the other hand, in a pioneer study, Scheller et $\mathrm{al}^{11}$ demonstrated that, in patients with BMS-ISR, DEB were superior to balloon angioplasty alone. In that study, no single patient in the DEB arm required repeated revascularisation during the first year. Another randomised trial demonstrated that DEB were superior to paclitaxel DES in this scenario ${ }^{12}$. All of these findings confirm that BMS-ISR can currently be treated effectively with both DES and DEB.

Conversely, patients with DES-ISR still remain a major therapeutic challenge ${ }^{3-6}$. The results of interventions in this setting are significantly poorer than those seen in patients treated for BMS-ISR ${ }^{5,6}$. However, the value of DES in patients with DESISR has been established ${ }^{10,21}$. In this scenario, many interventional cardiologists advocate the use of a "switch" DES strategy. Although indeed attractive, many retrospective observational studies yielded conflicting data in this regard ${ }^{3}$. In patients with sirolimus DES-ISR, the randomised ISAR-DESIRE 2 trial ${ }^{10}$ was unable to demonstrate the superiority of paclitaxel DES over sirolimus DES. It might be argued, however, that paclitaxel DES are less potent inhibitors of neointimal proliferation compared with sirolimus DES. Conversely, in the prospective multicentre RIBS III study ${ }^{21}$, the switch strategy was shown to be superior to other therapeutic modalities. Another major pending question is whether second-generation DES are indeed superior to firstgeneration DES in these patients. A sub-analysis of the RIBS III study $^{21}$ also supported this hypothesis. However, the potential value of second-generation DES in patients with DES-ISR warrants further investigation.

DEB constitute yet another attractive strategy for patients with DES-ISR $^{13-17}$. In patients with limus DES-ISR, a small, singlecentre, randomised study suggested that DEB were superior to conventional balloon angioplasty ${ }^{13}$. Similar findings were confirmed in a multicentre randomised trial in patients with any type of DES-ISR ${ }^{14}$. Recently, the ISAR-DESIRE 3 trial $^{17}$ allocated patients with limus DES-ISR to either DEB, paclitaxel DES or conventional balloon angioplasty. This randomised study demonstrated the non-inferiority of DEB compared with paclitaxel DES but also the superiority of these two strategies compared with balloon angioplasty ${ }^{17}$. However, in this study a loss of $0.37 \mathrm{~mm}$ was found in the DEB arm, which is much larger than that obtained with DEB in patients with BMS-ISR. Additional studies have confirmed that DEB are more effective in patients with BMS-ISR than in those with DES-ISR ${ }^{15}$. Finally, the PEPCAD China trial ${ }^{16}$ (220 patients with DES-ISR) demonstrated that the late lumen loss in the DEB arm $(0.46 \mathrm{~mm})$ was non-inferior to that found in the paclitaxel DES arm $(0.55 \mathrm{~mm})$. All of these studies confirm the relatively large late loss consistently seen after treatment of DES-ISR.

\section{Additional insights and rationale for the current study}

Overall, data from randomised trials suggest that DEB are superior to balloon angioplasty and equivalent to first-generation DES in patients with BMS-ISR or DES-ISR ${ }^{11-17}$. Nevertheless, there is scarce information on the value of DEB as compared with secondgeneration DES in patients with ISR. Very recently, the RIBS V randomised trial ${ }^{18}$ compared EES with DEB in patients with BMS-ISR. Minimal lumen diameter (also selected as primary endpoint) and percent diameter stenosis at follow-up were significantly superior in the EES arm. However, the angiographic late loss was very low and similar in both arms. Likewise, the binary restenosis rate was also very low (single digit figures) and equivalent in both arms. Last but not least, no differences were found in main clinical outcome measures, including target vessel revascularisation, at one year ${ }^{18}$.

From a technical standpoint, some final issues should be addressed. First, the use of therapies with profound antiproliferative properties may be complicated by edge effects. To avoid this problem, special care will be taken in this study to prevent geographic miss-related phenomena ${ }^{8,21}$. Second, intracoronary imaging may be able to unravel the underlying substrate of ISR and may help to optimise the results of these repeated procedures. The dedicated imaging substudies of RIBS IV will hopefully shed additional light on this issue. Finally, in all previous randomised trials on ISR only a particular DEB type (using iopromide as a hydrophilic spacer) was used ${ }^{11-18}$. The same DEB was selected in the present study, as a class effect cannot be anticipated for all available DEB.

\section{Conclusions}

This multicentre randomised clinical trial will compare the relative efficacy of EES and DEB for patients with DES-ISR.

\section{Impact on daily practice}

Treatment of DES-ISR still remains a technical and clinical challenge. The results of this trial will help to elucidate the safety and efficacy of DEB and EES (second-generation DES) in patients with DES-ISR. The results of this study will determine whether EES are able to obtain better late angiographic results than DEB in patients with DES-ISR. Furthermore, this study will also compare the late clinical outcome of these two therapeutic strategies (in particular, target vessel and target lesion revascularisation), thus providing new evidence to inform clinical practice.

\section{Funding}

There was an unrestricted grant from B. Braun Surgical and Abbott Vascular to support this study.

\section{Conflict of interest statement}

The authors have no conflicts of interest to declare.

\section{References}

1. Stefanini GG, Holmes DR Jr. Drug-eluting coronary-artery stents. N Engl J Med. 2013;368:254-65. 
2. García del Blanco B, Rumoroso Cuevas JR, HernandezHernandez F, Trillo-Nouche R. Spanish Cardiac Catheterization and Coronary Intervention Registry. 22nd official report of the Spanish Society of Cardiology Working Group on Cardiac Catheterization and Interventional Cardiology (1990-2012). Rev Esp Cardiol (Engl Ed). 2013;66:894-904.

3. Alfonso F, Byrne RA, Rivero F, Kastrati A. Current treatment of in-stent restenosis. J Am Coll Cardiol. 2014;63:2659-73.

4. Dangas GD, Claessen BE, Caixeta A, Sanidas EA, Mintz GS, Mehran R. In-stent restenosis in the drug-eluting stent era. $J$ Am Coll Cardiol. 2010;56;1897-907.

5. Steinberg DH, Gaglia MA Jr, Pinto Slottow TL, Roy P, Bonello L, De Labriolle A, Lemesle G, Torguson R, Kineshige K, Xue Z, Suddath WO, Kent KM, Satler LF, Pichard AD, Lindsay J, Waksman R. Outcome differences with the use of drug-eluting stents for the treatment of in-stent restenosis of bare-metal stents versus drug-eluting stents. Am J Cardiol. 2009;103;491-5.

6. Byrne RA, Cassese S, Windisch T, King LA, Joner M, Tada T, Mehilli J, Pache J, Kastrati A. Differential relative efficacy between drug-eluting stents in patients with bare metal and drug-eluting stent restenosis; evidence in support of drug resistance: insights from the ISAR-DESIRE and ISAR-DESIRE 2 trials. EuroIntervention. 2013;9:797-802.

7. Alfonso F, Zueco J, Cequier A, Mantilla R, Bethencourt A, López-Minguez JR, Angel J, Augé JM, Gómez-Recio M, Morís C, Seabra-Gomes R, Perez-Vizcayno MJ, Macaya C; Restenosis Intrastent: Balloon Angioplasty Versus Elective Stenting (RIBS) Investigators. A randomized comparison of repeat stenting with balloon angioplasty in patients with in-stent restenosis. J Am Coll Cardiol. 2003;42:796-805.

8. Alfonso F, Pérez-Vizcayno MJ, Hernandez R, Bethencourt A, Martí V, López-Mínguez JR, Angel J, Mantilla R, Morís C, CequierA, Sabaté M, Escaned J, Moreno R, Bañuelos C, Suárez A, Macaya C; RIBS-II Investigators. A randomized comparison of sirolimus-eluting stent with balloon angioplasty in patients with in-stent restenosis: results of the Restenosis Intrastent: Balloon Angioplasty Versus Elective Sirolimus-Eluting Stenting (RIBS-II) trial. J Am Coll Cardiol. 2006;47:2152-60.

9. Kastrati A, Mehilli J, von Beckerath N, Dibra A, Hausleiter J, Pache J, Schühlen H, Schmitt C, Dirschinger J, Schömig A; ISARDESIRE Study Investigators. Sirolimus-eluting stent or paclitaxeleluting stent vs balloon angioplasty for prevention of recurrences in patients with coronary in-stent restenosis: a randomized controlled trial. JAMA. 2005;293:165-71.

10. Mehilli J, Byrne RA, Tiroch K, Pinieck S, Schulz S, Kufner S, Massberg S, Laugwitz KL, Schömig A, Kastrati A; ISAR-DESIRE 2 Investigators. Randomized trial of paclitaxel- versus sirolimuseluting stents for treatment of coronary restenosis in sirolimuseluting stents: the ISAR-DESIRE 2 (Intracoronary Stenting and Angiographic Results: Drug Eluting Stents for In-Stent Restenosis 2) study. J Am Coll Cardiol. 2010;55:2710-6.

11. Scheller B, Hehrlein C, Bocksch W, Rutsch W, Haghi D, Dietz U, Böhm M, Speck U. Treatment of coronary in-stent restenosis with a paclitaxel-coated balloon catheter. $N$ Engl J Med. 2006;355:2113-24.

12. Unverdorben $M$, Vallbracht $C$, Cremers $B$, Heuer $H$, Hengstenberg C, Maikowski C, Werner GS, Antoni D, Kleber FX, Bocksch W, Leschke M, Ackermann H, Boxberger M, Speck U, Degenhardt R, Scheller B. Paclitaxel-coated balloon catheter versus paclitaxel-coated stent for the treatment of coronary in-stent restenosis. Circulation. 2009;119:2986-94.

13. Habara S, Mitsudo K, Kadota K, Goto T, Fujii S, Yamamoto H, Katoh H, Oka N, Fuku Y, Hosogi S, Hirono A, Maruo T, Tanaka H, Shigemoto Y, Hasegawa D, Tasaka H, Kusunose M, Otsuru S, Okamoto Y, Saito N, Tsujimoto Y, Eguchi H, Miyake K, Yoshino M. Effectiveness of paclitaxel-eluting balloon catheter in patients with sirolimus-eluting stent restenosis. JACC Cardiovasc Interv. 2011;4:149-54.

14. Rittger H, Brachmann J, Sinha AM, Waliszewski M, Ohlow M, Brugger A, Thiele H, Birkemeyer R, Kurowski V, Breithardt OA, Schmidt M, Zimmermann S, Lonke S, von Cranach M, Nguyen TV, Daniel WG, Wöhrle J. A randomized, multicenter, single-blinded trial comparing paclitaxel-coated balloon angioplasty with plain balloon angioplasty in drug-eluting stent restenosis: the PEPCADDES study. J Am Coll Cardiol. 2012;59:1377-82.

15. Habara S, Iwabuchi M, Inoue N, Nakamura S, Asano R, Nanto S, HayashiY, Shiode N, Saito S, IkariY, Kimura T, Hosokawa J, Nakamura M, Kotani J, Kozuma K, Mitsudo K. A multicenter randomized comparison of paclitaxel-coated balloon catheter with conventional balloon angioplasty in patients with bare-metal stent restenosis and drug-eluting stent restenosis. Am Heart J. 2013;166:527-33.

16. Xu B, Gao R, Wang J, Yang Y, Chen S, Liu B, Chen F, Li Z, Han Y, Fu G, Zhao Y, Ge J; PEPCAD China ISR Trial Investigators. A prospective, multicentre, randomized trial of paclitaxel-coated balloon versus paclitaxel-eluting stent for the treatment of instent restenosis: results from the PEPCAD China ISR trial. JACC Cardiovasc Interv. 2014;7:204-11.

17. Byrne RA, Neumann FJ, Mehilli J, Pinieck S, Wolff B, Tiroch K, Schulz S, Fusaro M, Ott I, Ibrahim T, Hausleiter J, Valina C, Pache J, Laugwitz KL, Massberg S, Kastrati A; ISAR-DESIRE 3 investigators. Paclitaxel-eluting balloons, paclitaxel-eluting stents, and balloon angioplasty in patients with restenosis after implantation of a drug-eluting stent (ISAR-DESIRE 3): a randomised, openlabel trial. Lancet. 2013;381:461-7.

18. Alfonso F, Pérez-Vizcayno MJ, Cárdenas A, García Del Blanco B, Seidelberger B, Iñiguez A, Gómez-Recio M, Masotti M, Velázquez MT, Sanchís J, García-Touchard A, Zueco J, Bethencourt A, Melgares R, Cequier A, Dominguez A, Mainar V, López-Mínguez JR, Moreu J, Martí V, Moreno R, JiménezQuevedo P, Gonzalo N, Fernández C, Macaya C; RIBS V Study Investigators, under the auspices of the Working Group on Interventional Cardiology of the Spanish Society of Cardiology. A randomized comparison of drug-eluting balloon versus everolimus-eluting stent in patients with bare-metal stent in-stent restenosis: the RIBS V Clinical Trial (Restenosis Intra-stent of Bare Metal 
Stents: paclitaxel-eluting balloon vs. everolimus-eluting stent). J Am Coll Cardiol. 2014;63:1378-86.

19. Alfonso F, Fernandez C. Second-generation drug-eluting stents. Moving the field forward. J Am Coll Cardiol. 2011;58:26-9.

20. Palmerini T, Biondi-Zoccai G, Della Riva D, Stettler C, Sangiorgi D, D'Ascenzo F, Kimura T, Briguori C, Sabatè M, Kim HS, De Waha A, Kedhi E, Smits PC, Kaiser C, Sardella G, Marullo A, Kirtane AJ, Leon MB, Stone GW. Stent thrombosis with drug-eluting and bare-metal stents: evidence from a comprehensive network meta-analysis. Lancet. 2012;379:1393-402.

21. Alfonso F, Pérez-Vizcayno MJ, Dutary J, Zueco J, Cequier A, García-TouchardA, Martí V, Lozano I, Angel J,HernándezJM, LópezMínguez JR, Melgares R, Moreno R, Seidelberger B, Fernández C, Hernandez R; RIBS-III Study Investigators (under the auspices of the Working Group on Interventional Cardiology of the Spanish Society of Cardiology). Implantation of a drug-eluting stent with a different drug (switch strategy) in patients with drug-eluting stent restenosis. Results from a prospective multicenter study (RIBS III [Restenosis Intra-Stent: Balloon Angioplasty Versus Drug-Eluting Stent]). JACC Cardiovasc Interv. 2012;5:728-37.

22. Cutlip DE, Windecker S, Mehran R, Boam A, Cohen DJ, van Es GA, Steg PG, Morel MA, Mauri L, Vranckx P, McFadden E, Lansky A, Hamon M, Krucoff MW, Serruys PW; Academic Research Consortium. Clinical end points in coronary stent trials: a case for standardized definitions. Circulation. 2007;115:2344-51.
23. Mehran R, Dangas G, Abizaid AS, Mintz GS, Lansky AJ, Satler LF, Pichard AD, Kent KM, Stone GW, Leon MB. Angiographic patterns of in-stent restenosis: classification and implications for long-term outcome. Circulation. 1999;100:1872-8.

24. Alfonso F, Cequier A, Angel J, Martí V, Zueco J, Bethencourt A, Mantilla R, López-Minguez JR, Gómez-Recio M, Morís C, Perez-Vizcayno MJ, Fernández C, Macaya C, SeabraGomes R; Restenosis Intra-stent Balloon angioplasty versus elective Stenting (RIBS) Investigators. Value of the American College of Cardiology/American Heart Association angiographic classification of coronary lesion morphology in patients with in-stent restenosis. Insights from the Restenosis Intra-stent Balloon angioplasty versus elective Stenting (RIBS) randomized trial. Am Heart J. 2006;151:681.e1-681.e9.

25. Alfonso F, García P, Fleites H, Pimentel G, Sabaté M, Hernández R, Escaned J, Bañuelos C, Pérez-Vizcayno MJ, Moreno R, Macaya C. Repeat stenting for the prevention of the early lumen loss phenomenon in patients with in-stent restenosis. Angiographic and intravascular ultrasound findings of a randomized study. Am Heart J. 2005;149:e1-8.

\section{Online data supplement}

Online Appendix. The Restenosis Intra-stent: drug-eluting Balloon vs. everolimus-eluting Stent (RIBS IV) study investigators, coordinators and sites. 


\section{Online data supplement}

Online Appendix. The Restenosis Intra-stent: drug-eluting Balloon vs. everolimus-eluting Stent (RIBS IV) study investigators, coordinators and sites are as follows:

Coordinating centre: Hospital Universitario Clínico San Carlos, Madrid, Spain

Steering Committee: F. Alfonso (Chairman and Principal Investigator), J. Zueco, A. Cequier, C. Morís, C. Macaya

Clinical Events Committee: R. Hernández, M. Sabaté

Coronary Angiography Core Laboratory (Hospital Universitario Clínico San Carlos, Madrid, Spain): A. Cárdenas, M.J. Pérez-Vizcayno

Data Coordination, Safety Monitoring and Statistical Committee: M.J. Pérez-Vizcayno, C. Fernández-Pérez

Intravascular Ultrasound and Optical Coherence Tomography Committee: N. Gonzalo, F. Alfonso

\section{Sites and Investigators:}

1. Hospital Universitario Clínico San Carlos, Madrid, Spain (P. Jiménez-Quevedo, N. Gonzalo, J. Escaned, A. FernándezOrtiz, C. Macaya);

2. Hospital Universitario Vall d'Hebron, Barcelona, Spain (B. García del Blanco);

3. Hospital Universitario de la Princesa, Madrid, Spain (F. Alfonso, A. Benedicto, F. Rivero);

4. Complejo Hospitalario Universitario de Vigo, Vigo, Spain (A. Iñiguez);

5. Hospital Universitario de Torrecárdenas, Almería, Spain (M. Gómez-Recio);

6. Hospital Universitario Clinic de Barcelona, Barcelona, Spain (M. Masotti);

7. Hospital Universitario 12 de Octubre, Madrid, Spain (T. Velázquez);

8. Hospital Universitario Clínico de Valencia, Valencia, Spain (J. Sanchís);

9. Hospital Universitario Puerta de Hierro, Majadahonda, Spain (A. García-Touchard);

10. Hospital Universitario Marqués de Valdecilla, Santander, Spain (J. Zueco);
11. Hospital Universitario Son Espases, Palma de Mallorca, Spain (A. Bethencourt);

12. Hospital Universitario Virgen de las Nieves, Granada, Spain (R. Melgares);

13. Hospital Universitario de Bellvitge, Barcelona, Spain (A. Cequier);

14. Hospital Universitario Virgen de la Victoria, Málaga, Spain (J.M. Hernández);

15. Hospital Universitario de Alicante, Alicante, Spain (V. Mainar);

16. Hospital Universitario Infanta Cristina, Badajoz, Spain (J.R. López-Mínguez);

17. Hospital Universitario Virgen de la Salud, Toledo, Spain (J. Moreu);

18. Hospital Universitario Sant Pau, Barcelona, Spain (V. Martí);

19. Hospital Universitario La Paz, Madrid, Spain (R. Moreno);

20. Hospital Universitario General de Valencia, Valencia, Spain (F. Pomar);

21. Complejo Hospitalario de Navarra, Pamplona, Spain (R. Lezaún);

22. Hospital Universitario Carlos Haya, Málaga, Spain (C.A. Urbano-Carrillo);

23. Hospital Universitario Miguel Servet, Zaragoza, Spain (M.C. Ferrer-García);

24. Complejo Hospitalario Universitario de A Coruña, La Coruña, Spain (N. Vázquez);

25. Hospital Universitario Central de Asturias, Oviedo, Spain (C. Morís).

The study will be conducted under the auspices of the Working Group of Coronary Interventions of the Spanish Society of Cardiology (Sociedad Española de Cardiología). This trial is an investigator-driven initiative. Data monitoring, entrance into the database, database maintenance, general and statistical analyses and drafting and submission of the final manuscript will be performed by the investigators. There is an unrestricted grant from B. Braun Surgical and Abbott Vascular to support this study. 